\title{
Study of Safe Sensor Configurations in Human Robot Collaboration
}

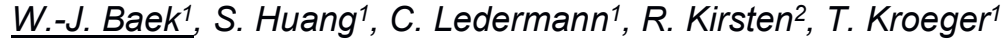 \\ ${ }^{1}$ Karlsruhe Institute of Technology (KIT), Institute of Anthropomatics and Robotics - Intelligent Process \\ Automation and Robotics (IAR-IPR), Engler-Bunte-Ring 8, 76131 Karlsruhe, Germany \\ ${ }^{2}$ ABB AG Forschungszentrum, Wallstadter Straße 59, 68526 Ladenburg, Germany
}

\begin{abstract}
In this contribution, we present two approaches yielding an automatic generation of the optimal sensor configuration in a defined human robot collaboration scenario with respect to the compliance of the human operator's safety and the minimum floor space consumption in the working cell. Based on a simulation setup created with the software Gazebo 7, we develop two algorithms providing the combination and position information of such devices. Following the derived procedures, we focus not only on the placement of safety elements, namely the laser scanner, fences and safety mats but also the influence of the corresponding parameters on the obtained results.
\end{abstract}

Keywords: Sensor Placement, Safety, Human Robot Collaboration.

\section{Introduction}

The deployment of robots in industrial applications has led to promising results in recent years, in particular in manufacturing facilities. Typically, robots are made responsible for simple tasks including welding, picking and placing or even packaging and labeling products. One of the most important aspects for human operators involved in such processes is the compliance of safety, which is basically defined by norms provided by the International Organization of Standardization (ISO). In order to realize a safe working environment, manufacturing facilities make use of safety elements like mechanical barrier elements or sensors. Doing so, the working cell will be monitored and human operators are supposed to be alerted in time when entering a critical zone around the robot. Robots on the other hand are supposed to slow down or stop their movement accordingly.

One crucial issue in this context is the optimal placement of safety devices in the human robot working cell. Within the scope of this study, two approaches yielding the automatic generation of localization points for safety elements have been developed. Based on the software Gazebo, a simulation environment representing a human robot collaboration scenario has been established. By defining a certain area around the industrial robot, we investigate how sensors or mechanical barrier elements have to be positioned in order to slow down, stop the robot movement or prevent the human being from entering the working area of the robot, thus preventing the human being from hazards. The distinction of critical and safe situations is achieved on norms defined by the International Organization for Standardization (ISO).

\section{State of the Art}

In previous works, such as (Andreas Krause, 2006), (Emily Clark, 2018), (Zainab M. Ismail, 2018), the sensor placement issue has been dealt in the context of different applications as structural health monitoring and computer communication networks. In particular, (Andreas Krause, 2006) presents an algorithm providing sensor localization points based on probabilistic models aiming cost-effective and informative selections.

Our contribution focuses on the determination of a combination of devices yielding the compliance of safety for the human operator. To date, there exist different norms and measures defined by the International Organization of Standardization, which are usually considered as criteria for safety. Apart from this fact the movement of the robot in human robot cooperation, is distinguished in three categories according by IEC 50204-1. ISO norm 13855 has been stated in 2010 and equals a simple relation which allows the determination of a safety distance $S$ between 
the actuators for a known velocity of the approaching human operator $v$ and different influences addressed by the constant $c$ :

$$
S=v t+c
$$

A more specified formalization is presented by norm ISO/TS 15066 and a publication Marvel et al., 2016 (Jeremy A. Marvel, 2017), where $\mathrm{V}_{\mathrm{H}}$ refers to the velocity of the human operator, $V_{R}$ to such of the robot and $v_{s}$ considers the velocity of sensors:

$$
\begin{aligned}
& S\left(t_{0}\right) \geq \int_{\tau=0}^{\tau=t_{0}+T_{R}+T_{S}} v_{H}(\tau) d \tau+\int_{\tau=t_{0}}^{\tau=t_{0}+T_{R}} v_{R}(\tau) d \tau \\
& +\int_{\tau=t_{0}+T_{R}}^{\tau=t_{0}+T_{R}+T_{S}} v_{S}(\tau) d \tau+C+Z_{S}+Z_{R}
\end{aligned}
$$

Here, $T_{R}$ denotes the time of robot manipulator needed to respond, $T_{S}$ the time required to bring the robot to a stop, $\mathrm{C}$ the intrusion distance safety margin based on the expected reach of the human, $Z_{s}$ the sensor position uncertainty and $Z_{R}$ the robot's position uncertainty. In this study, sensors are assumed to be positioned and not moved afterwards. In addition, uncertainties are not part of investigation. Thus, the last term vanishes. Furthermore, the approaches apply to cases where the robot and the human operator move with constant velocities, i.e. it is assumed that the actuators are not subject to any forces which might occur in the setup. Consequently, equation (2) becomes equation (1). In order to distinguish safe from critical situations, we thus refer to equation (1) in the presented approaches.

\section{Simulation procedure}

Investigations within the scope of this contribution have been performed based on simulations conducted with the open source software ROS (Robot Operating System) kinetic and Gazebo 7. Combining ROS with Gazebo enabled the consideration of dynamic states of the actuators. In the scope of this study, ROS kinetic has been applied on the simulation software Gazebo. The considered setup including two tables is shown in figure 1.

\section{Simulation steps}

The focus of the simulations performed lies in the determination of the optimal sensor placement with respect to two constraints in the given order:
1) Safety according to norm ISO 15066

2) Minimum consumed floor space by devices

In the following, the cycle of one simulation run will be explained.

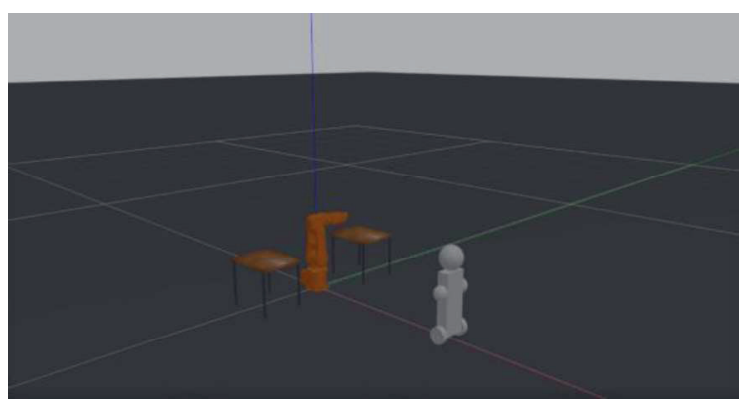

Figure 1: Screenshot of the simulation scenario in Gazebo 7. The simulation setup consists of two tables and the robot model ABB-IRB120 moving its arms with constant velocity. The human model performs random trajectories within the working cell.

\section{a) Placement of safety element:}

The first step in the simulation procedure is given by the placement of the safety device. In the first loop, the element type and its position are selected randomly. Subsequent steps depend on the approach applied. Within the frame of this contribution, two methods have been considered: While the first method relies on static parameters of the safety devices, the second algorithm allows the variation of the measures. Both approaches will be explained in more detail in IV.

\section{b) Classification of situations in safe and} critical: Within the simulation procedure, different scenarios are expected to occur which can be classified in safe and critical. In first place, this classification is based on the different radii around the robot model representing different zones with respect to the human operator's safety as shown in figure 2 . Once the human operator approaches the robot and enters one of the circles, the situation is categorized as critical. In our contribution, we assume the outermost circle as the measure for safety. Doing so, we argue that the worst case scenarios are covered in our investigations.

c) Generation of sensor localization points: The result obtained in the previous step equals a binary output distinguishing safe from critical situations. In case the binary output links to the occurrence of a critical situation, the second 
algorithm is initialized. This algorithm is responsible for the generation of the sensor position. Once generated, the information is forwarded to the simulation interface in order to visualize the sensor type and its location.

d) Termination of simulation: If the binary output of the external observer in c) categorizes the situation as safe, the simulation is terminated. The location information as well as the type of the sensors placed in the simulation and the corresponding floor space consumption is stored in an array.

\section{e) Selection of the combination yielding the} minimum floor space:

After termination, the combination of safety elements including the corresponding consumed floor space is stored in an array. The entries of this array are sorted with respect to the consumed floor space values for each combination of devices.

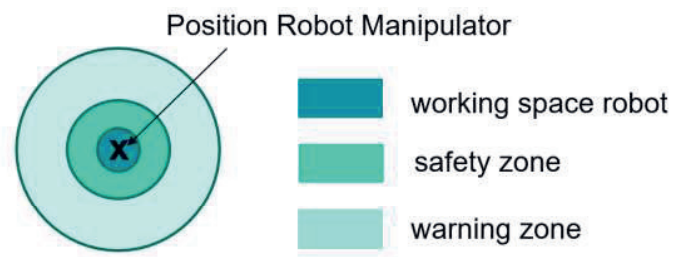

Figure 2: Depiction of the warning zones around the robot. Considering the position of the robot in the center, the zones around it can be split into three different zones as shown above.

\section{Considered Devices}

As mentioned above, three different safety devices have been subject to investigations. Besides laser scanners and safety mats, we make use of one mechanical barrier element to investigate its influence on the result. If not stated specifically, the characteristics of such are set according to the following table.

Table 1: Overview of considered devices

\begin{tabular}{|l|l|l|l|}
\hline & $\begin{array}{l}\text { Laser } \\
\text { scanner }\end{array}$ & $\begin{array}{l}\text { Safety } \\
\text { Mat }\end{array}$ & $\begin{array}{l}\text { Fence } \\
\text { Element }\end{array}$ \\
\hline \multirow{5}{*}{ Measures } & $\begin{array}{l}\text { Aperture } \\
\text { angle: } \\
120^{\circ}\end{array}$ & $\begin{array}{l}\text { Width: } \\
0.5 \mathrm{~m}\end{array}$ & $\begin{array}{l}\text { Thickness: } \\
0.1 \mathrm{~m}\end{array}$ \\
\cline { 2 - 4 } & $\begin{array}{l}\text { Range: } \\
7.0 \mathrm{~m}\end{array}$ & $\begin{array}{l}\text { Length: } \\
0.5 \mathrm{~m}\end{array}$ & $\begin{array}{l}\text { Length: } \\
1.0 \mathrm{~m}\end{array}$ \\
\hline
\end{tabular}

\section{Approaches}

In both algorithms presented below, above devices have been taken into account. However, the methods are distinguished by the fact that one relies only on the localization issue taking the measures of the devices as fixed values while the second algorithm considers different values for each parameter, thereby allowing for the investigation of the measures' influences on the recommended combination of devices.

\section{Customized Approach from scratch}

The first approach has been developed from scratch based on three main assumptions, which are defined in the following.

Assumption 1: Sensor characteristics are given and set according to table 1 . The specifications are known and do not have to be evaluated. Therefore, device parameters are treated as constant parameters in this approach.

Assumption 2: Once a sensor or barrier element is placed, the corresponding area is covered immediately, i.e. the latency time of the sensor $t_{s, \text { latency }}$ is neglected assuming:

$$
t_{s, \text { latency }}=0 s \text {. }
$$

Assumption 3: If a sensor or barrier element is placed, it covers and detects the relevant area. The direction of the coverage is therefore assumed to be positioned ideally.

The classification of safe and critical situations is executed by an external code, the so-called classification code, which evaluates the situation as follows: By means of the sensor characteristics, which are basically the radius of the range $R_{S}$ covered by it and its aperture angle $\varphi_{\mathrm{s}}$, the covered area $A_{S}$ can be determined with the following relation:

$$
A_{S}=\int_{0}^{R_{S}} \int_{0}^{\varphi_{S}} r d \varphi d r
$$

On the other hand, the area corresponding to the outermost zone of the robot manipulator $A_{R}$ can be simply calculated via

$$
A_{R}=\pi \cdot r_{w, \max }^{2}
$$

where $r_{w, \max }$ denotes the radius of the warning zone. Here, the worst case is taken into account, i.e. the widest range which can be 
reached by the robot and therefore the largest zone is considered.

Hence, the warning zone corresponding to the robot can be assumed to be static.

As the exact position of the sensor is known, it is possible to estimate if there is a cut set of

$A_{S}$ and $A_{R}$ by means of equation (6).

$$
A_{S} \cap A_{R} \neq 0
$$

i.e. if the detection area of the sensor covers the outer circle of the robot's warning zone as depicted in figure 2 .

In order to simplify this issue, it is possible to only consider the circle of the warning zone. Doing so, the classification still yields an output corresponding to the worst case scenario. Hence, the prevention of the human operator from hazards due to the robot is assumed to be fulfilled with a high probability.

One possible approach to face this issue is the representation of the warning zone by means of equidistant discrete points. By verifying how many of such points are covered by the detection area of the sensor, it can be estimated if the placement of additional safety element is required, that is, if the loop will be terminated. The stepwise addition of sensors is carried out until the coverage of the whole circle is reached. Once all points have been covered by sensors, the sensor placement loop is terminated.

In contrast to sensors, mechanical barrier elements do not offer a certain aperture angle. Hence, such barrier elements are treated as lines, projecting the endpoint to the circle and thus determining the area covered of the warning zone- In terms of the location of the sensors to be placed, a method which contains a strict structure is applied in this approach. In general, the type of the safety element is chosen randomly at the beginning of each loop. Also, the position of the first safety element is generated randomly. If the corresponding area $A_{S}$ does not cover all points, i.e. if it does not fulfill the conditions of safety, the start of an additional loop is initialized. The second safety element then is localized by reflecting the position of the first element on a line.

Before placing the safety device on the calculated position, it is checked if a previous element already has been positioned on the same point. If this applies, two further lines which are perpendicular to each other are defined and act as mirror lines.

In case the safety requirements are not fulfilled after having completed the reflection process on each line, additional sensors are placed by calculating half of the distance to the neighboring element.

Once a simulation run is terminated, the corresponding data, namely the number and types of safety elements is stored in an array. In addition, the floor space covered by all sensors is calculated which can be completed because sensor parameters are fully known. Doing so, the combination yielding the minimal floor space can be determined by comparing the calculated values.

The comparison of the obtained results with regard to the consumed floor space can be performed either automatically or manually by the user, depending on the number of sensors to be considered.

In the presented approach, the number of considered sensor types is limited.

Thus, there exist a fixed number of possible solutions. Presented approaches therefore are recommended for applications where only small number of safety elements is taken into account.

\section{Approach based on MatLab}

In contrast to the previous method, the second approach relies on the usage of the software MatLab. Using this tool, the workspace of the human operator and the robot was split in different grids of the size of $0.2 \mathrm{~m} \times 0.2 \mathrm{~m}$. By considering different assumptions, the device parameters, such as the aperture angle, width and lengths have been varied to determine the most reasonable combination of safety elements.

Apart from that, comparisons between the results obtained in approach 1 allows for the determination of the dimensions of employed devices with respect to the minimum floor space coverage. The second assumption referring to a vanishing latency time of safety elements was taken accordingly. The main difference between the two approaches therefore lies in the fact that the previous method takes sensor specifications as constant values while this approach considers different measures.

\section{Results and Discussion}

Figure 3 presents the consumed floor space required for certain sets of sensors fulfilling the safety constraint after applying the first approach presented above. The $\mathrm{x}$-axis shows the space area taken into account by a certain combination of safety elements. The number of the recommended elements is shown on the $y$ axis. Such a representation is possible and 
representative due to the fact that the combination of safety elements is not ambiguous with regard to the floor space consumed, that is, to each value of consumed floor space one combination has been selected by the algorithm. Figure 3 thus allows to obtain the recommended combination of devices for a given floor space. For example, the requirement of safety and minimum floor space consumption in consideration of the given safety elements, can be achieved by making use of two laser scanners which result in a consumed floor space of

$$
A_{\text {opt }}=0.0575 \mathrm{~m}^{2} \text {. }
$$

Apart from this fact, it can be seen that the amount of floor space is highly correlated to the number of safety mats. In contrast, the number of laser scanners and fence elements do not show clearly distinguishable courses over the area. This observation matches with the theoretical expectancy since safety mats correspond to the largest area among the three devices considered in this study. In addition, it is noted that merely using the safety mats cannot yield a safe configuration. In case 13 mats are placed in the working cell, at least one laser scanner is required to guarantee the human operator's safety. This result can be explained by the fact that the area covered by the mats equals to the floor space taken up. Since the dimensions of the safety mats show the same value for the length as well as for the width, i.e. are considered as quadratic elements, the consumed floor space increases quadratically while the covered area of the circle representing the warning zone arises with linear behavior. However, based on the obtained results, it is recommended to employ two laser scanners at two opposite corners of the working cell. Such a scenario is depicted in figure 4 where the blue area corresponds to the space detected by the laser scanners.

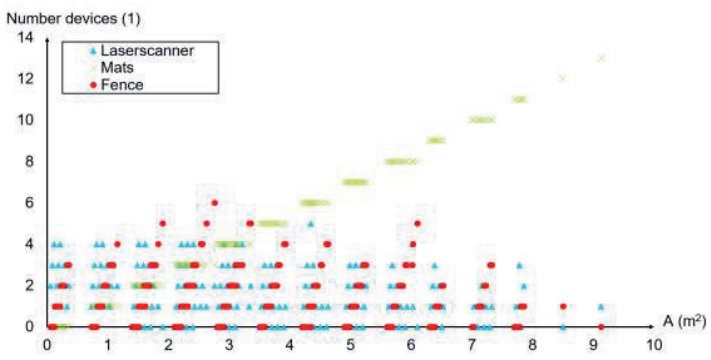

Figure 3: Evaluation of different combinations of safety elements over the consumed floor space based on the elements parameters as presented by table 1. For a given floor space, the optimal combination of safety elements can be determined by this figure.



Figure 4: Setup depicted in Gazebo matching safety requirements and minimum floor space consumption constraints. This configuration includes two laser scanners which are positioned at two opposite corners. The blue area depicts the detection area of the devices.

\section{Variation of the parameters}

By making use of the second approach in MatLab, parameters of the considered devices have been varied. Doing so, it was investigated how such variations affect the overall results. Hence, this treatment refers to an optimization method in contrast to the previous approach. In the following, the results will be discussed in more detail. It is noted that only one parameter was subject to variation to each point of time while holding the parameters of the remaining elements constant in order to investigate the influence respectively.

a) Variation of the Range (Laser Scanner) One characteristic of the laser scanner lies in the fact that two parameters can be varied. In first place, the variation of its range has been performed.

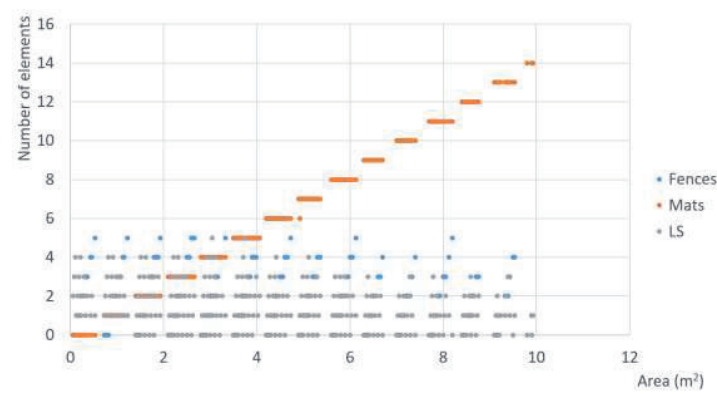

Figure 5: Number of safety elements over the consumed floor space for a laser scanner with a range of $5 \mathrm{~m}$ and an aperture angle of $120^{\circ}$. As figure 5 shows, the number of required devices increases with decreasing range values. At the same time the recommended number of fence elements shows a different behavior than in figure 3 .

Comparing this observation with the observation in figure 3 allows the conclusion that in above case the usage of a higher number of fence elements corresponds to a 
lower floor space consumption. According to figure 5 , the number of recommended number of mats in contrast is not affected by the variation of the laser scanner range.

\section{b) Variation of the Aperture Angle (Laser Scanner)}

In the subsequent step, the variation of the aperture angle has been performed.

Considering three different values for this quantity enables for the determination of its impact on the overall result. As shown in Appendix A., the deployment of fence elements with the dimensions of $0.1 \mathrm{~m} \times 1.0$ results in a smaller floor space consumption when considering smaller aperture angles of the laser scanner.

\section{c) Variation of the Fence Dimensions} Varying the dimensions of the fence leads to the finding that the effect of the laser scanner's parameters is strongly correlated with the number of recommended fences to achieve a minimum floor space consumption. This observation can be explained by the fact that safety mats show a quadratic behavior in context to the floor space consumption. Thus, the developed algorithms prefer the deployment of fences which result in the coverage of the warning zone. However, the correlation between the laser scanner and fence element is suggested to be investigated in more detail.

\section{d) Variation of the Safety Mat dimension} Changing the values of the mat's measures, where the width and length have been varied simultaneously have led to the conclusion that making use of mats is inefficient with respect to the achievement of a solution with the minimum floor space consumption. The largest dimension corresponds to a mat with dimensions of $1.5 \mathrm{~m}$ for each, width and height. However, the fulfillment of the safety constraint requires the employment of further devices. In addition, the amount of the required floor space increases rapidly with the number of employed safety mats. As a consequence, the usage of this device is not recommended at first glance.

However, presented approaches are based on the procedure of solely covering a circle corresponding to the outermost warning zone around a robot. In order to provide a further developed result, a more specified application scenario is needed. The variation of the safety mat parameters however do not lead to a different result with respect to the recommended combination regarding the minimum consumed floor space value.
Considering the simplified scenario described in figure 1 , the reduction of the laser scanners aperture angle or its range do not affect the results obtained through the algorithms.

Therefore, it can be stated that the employment of laser scanners is recommended independent from the variation of the safety elements parameters considered in our approach.

\section{Conclusion and Outlook}

In this contribution, we investigated the sensor placement issue in a human robot collaboration scenario by means of a simulation setup. It was aimed to fulfill two constraints, namely the human operator's safety defined by norm ISO 15066 in the first and the minimum floor space consumption in second place. We developed two approaches, leading to the same result for the considered scenario. Investigations concerning the influence of the safety devices specifications on the results showed that the deployment of two laser scanners with an aperture angle of $120^{\circ}$ and a range of $7 \mathrm{~m}$ is recommended for the considered scenario. The results have been obtained by means of two different methods. The first approach is based on an algorithm clearly defining the characteristics and certain measures before starting the simulation. In contrast, the focus of the second method was laid on the selection of the devices with most appropriate measures with regard to floor space consumption and safety. In both cases, safety was considered based on the norm ISO15066 taking into account the distance between robot and human. However, in order to derive a result applicable for real world applications, the human robot collaboration scenario has to be specified in more detail in order to meet the appropriate requirements. This would allow for the consideration of further additional aspects such as the throughput and economical costs. The result obtained by employing our algorithms yield the recommendation of using two laser scanners which matches the theoretical expectancy for the considered scenario. Merely considering the circle around the outer warning zone simplifies the issue such that velocity measures of the human operator are neglected. Therefore, it is highly recommended to develop further approaches, taking into account the priority of constraints, for example by means of weight factors or functions. Apart from this fact, the movement of the human operator could be part of further investigations, in terms of the direction. Body parts could be specified to distinguish the 
possible consequences of hazards on the human being. Similar to the consideration of different constraints in a certain order, one possible approach to treat this issue could be the use of an optimization method with weights.

\section{Appendix}

A. Variation of the Aperture Angle (Laser Scanner)

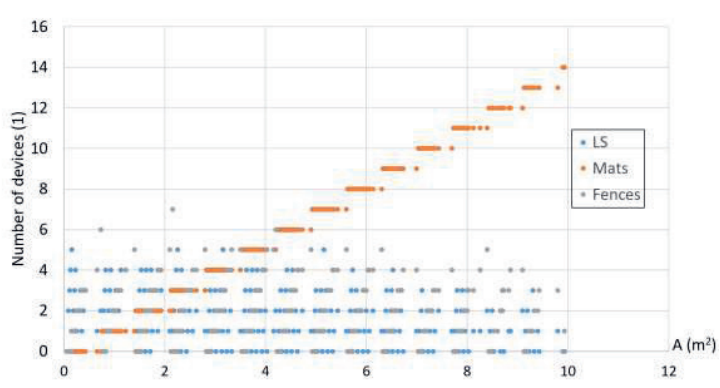

Figure 6: Recommended combination of safety devices for a laser scanner with an aperture angle of $45^{\circ}$. The recommended combination of safety devices is depicted over the required floor space.

\section{Literature References}

Andreas Krause, C. G. (2006). Near-optimal Sensor Placements: Maximizing Information while Minimizing Communication Cost. IPSN , p. 10.

Emily Clark, T. A. (2018). Greedy Sensor Placement with Cost Constraints. ArXiv Submission, p. 13.

Jeremy A. Marvel, R. N. (2017, August). Implementing speed and separation monitoring in collaborative robot. Robotics and ComputerIntegrated Manufacturing , pp. 144-155.

Zainab M. Ismail, M. A. (2018, June). The application of Genetic Algorithm for sensor placement of PZT wafers towards the application in structural health monitoring. European Conference on Non-Destructive Testing (ECNDT 2018), p. 9. 